\title{
Mass action in two-sex population models: encounters, mating encounters and the associated numerical correction
}

\author{
Katherine Snyder, Brynja Kohler and Luis F. Gordillo \\ Department of Mathematics and Statistics, Utah State University, Logan, UT, USA
}

\begin{abstract}
Ideal gas models are a paradigm used in Biology for the phenomenological modelling of encounters between individuals of different types. These models have been used to approximate encounter rates given densities, velocities and distance within which an encounter certainly occurs. When using mass action in two-sex populations, however, it is necessary to recognize the difference between encounters and mating encounters. While the former refers in general to the (possibly simultaneous) collisions between particles, the latter represents pair formation that will produce offspring. The classical formulation of the law of mass action does not account this difference. In this short paper, we present an alternative derivation of the law of mass action that uses dimensional reduction together with simulated data. This straightforward approach allows to correct the expression for the rate of mating encounters between individuals in a two-sex population with relative ease. In addition, variability in mating encounter rates (due to environmental stochasticity) is numerically explored through random fluctuations on the new mass action proportionality constant. The simulations show how the conditioned time to extinction in a population subject to a reproductive Allee effect is affected.
\end{abstract}

\section{ARTICLE HISTORY}

Received 31 October 2016

Accepted 28 February 2017

\section{KEYWORDS}

Mass action; dimensional analysis; environmental stochasticity

\section{Introduction}

In chemistry, the law of mass action states that the rate of a reaction is proportional to the product of the concentrations of the reactants. Since Alfred Lotka pioneered its use in Lotka (1925) to justify the encounter term in his predator-prey system of differential equations, the law has become ubiquitous in mathematical ecology for modelling the interactions between individuals of different groups. Lotka's arguments for the use of mass action in biological encounters were motivated by the analogy to the kinetic theory of gases (ideal gas models) and have been applied to describe a variety of phenomena, including fertilization kinetics, search theory and mate finding, see Hutchinson and Waser (2007), Voit, Martens, and Omholt (2015) for thorough reviews. Before Lotka, the chemical law of mass action was used for the first time by A.G. McKendrick for describing the interactions

CONTACT Luis F.Gordillo Luis.Gordillo@usu.edu

(c) 2017 The Author(s). Published by Informa UK Limited, trading as Taylor \& Francis Group.

This is an Open Access article distributed under the terms of the Creative Commons Attribution License (http://creativecommons.org/ licenses/by/4.0/), which permits unrestricted use, distribution, and reproduction in any medium, provided the original work is properly cited. 
among susceptible and infected individuals in epidemiological contexts (Heesterbeek, 2005; McKendrick, 1912).

In the theory of molecular collisions in gases, the collision frequency among particles of different types is expressed in terms of the velocities and radiuses of particles. The quantitative law is deduced directly from geometrical abstractions using the mean of a Poisson process that models the number of collisions a particle receives from others and where simultaneous collisions are allowed (Hutchinson \& Waser, 2007; Kauzmann, 2012). At relatively low population densities, the same idea is used as a phenomenological approach to approximate the encounter rates between males and females, with the birth rate taken proportional to the product of their densities (Bazykin, 1998; Fauvergue, 2013). We remark that two-sex population models become relevant when sexual dimorphism in vital rates is present, which has been observed in several species (Caswell, 2001).

While ideal gas models, which assume a heterogeneous population of linearly moving individuals, are used for modelling encounters of individuals, dispersal and movement of homogeneous populations in ecology are modelled following diffusion models, i.e. where individuals move randomly in space (Codling, Plank, \& Benhamou, 2008; Turchin, 1998). However, due to the complexity involved, most of the studies that include encounters in models of randomly moving organisms are based on computer simulations (see for instance Bartumeous, Catalan, Viswanathan, Raposo, \& da Luz, 2008; Gurarie \& Ovaskainen, 2011; James, Plank, and Brown, 2008, 2010, where encounters are defined in general animal search problems). This paper addresses the modelling of encounters that lead to reproduction, which requires ruling out simultaneous encounters that occur in the ideal gas model, i.e. encounters where a single female mates two or more males simultaneously, otherwise the rate at which new offspring appear would be overestimated. Thus, we differentiate between counting encounters, possibly simultaneous and counting mating encounters, which are understood here as the formation of female-male pairs from which offspring are successfully produced.

Our aim here is to present a correction for the constant used in the mass action term corresponding to the ideal gas model that accounts for the pair formation. To achieve this goal, we first build a functional relation among the variables using dimensional reduction and simulated data of individuals' movement. This allows us to approximate the value of the proportionality constant for the mass action with relative precision in comparison to theoretical results. Then, we generate new data through computer simulations that only count female-male pairs, and with this we approximate the new value for the constant. Finally, we use the new constant in the mass action law to explore the effects of environmental stochasticity on the conditioned time to extinction for a population model via the variability on encounter rates. The stochastic model is derived from a deterministic model that uses mass action at low population densities and thus shows a reproductive Allee effect (Courchamp, Berec, \& Gascoigne, 2008). The simulations reveal the effects that simultaneous random fluctuations around the new constant (and therefore the mating encounter rate) and demographic stochasticity have on the extinction time. This elementary example justifies having reasonable approximations for the non-linear term that models encounters: it demonstrates how variability in the environment could play an essential role in regulating the time to extinction distribution. 


\section{Dimensional reduction for mass action}

We consider a two-sex population and assume that individuals' movement is done in two spatial dimensions, i.e. we assume that the total variation in the displacement of each individual on the plane is much larger than changes made in their altitude. We also assume that individuals of both sexes are initially homogeneously mixed and uniformly distributed in space, and move over a terrain with area $A$ under the following assumptions:

- The velocity, $v$, is constant and the same for both sexes,

- Individuals move independently from each other,

- Movement is in straight lines,

- The initial direction of movement for each individual is independently chosen at random from the interval $[0,2 \pi)$,

- Individuals' sizes are negligible.

Let $n_{m}$ and $n_{f}$ be the number of males and females, respectively, and $c$ the average number of encounters that one female has with males during the observation time $t$. We assume that $c$ is related to (i) the velocity $v$, (ii) the density of males $n_{m} / A$ and (iii) the size of a small area surrounding the female where males are attracted to mate. This is thought as a circular area with radius $R$. We remark that these assumptions are the same used in the theory of molecular collisions to deduce the law of mass action for gases of two different types, with just the words 'particle' replacing 'individual' and 'type' instead of 'sex' (Hutchinson \& Waser, 2007; Kauzmann, 2012). We write the relation between the system parameters in terms of some (unknown) function $F$,

$$
c=F\left(\frac{n_{m}}{A}, v, t, R\right) .
$$

By the П-Theorem, see Barenblatt (2006) or Logan (2006) for instance, Equation (1) is equivalent to a relation that involves only the dimensionless quantities

$$
\Pi_{1}=\frac{v t R n_{m}}{A} \quad \text { and } \quad \Pi_{2}=\frac{v t}{R},
$$

that is,

$$
c=f\left(\frac{v t R n_{m}}{A}, \frac{v t}{R}\right),
$$

with $f$ yet to be determined.

Next, we use data generated from agent-based simulations that count the number of contacts (with males) per female. For the simulations, the individuals were programmed to follow the rules stated above and, for a single female, we counted a contact when its distance to a male is less than $R$. In the computations, the units chosen for length and time were meters and hours ( $1 \mathrm{~h}=1$ time step). We used fixed values for the time of observation, $t=t_{*}=24(\mathrm{~h})$, the radius $R=R_{*}=.05(\mathrm{~m})$ and the number of males, $n_{m *}=100$, while varying area size $A$ and individuals' velocity $v$. On the plane $v-A$, we arbitrarily choose the strip $\left[25,4 \times 10^{4}\right] \times\left[25,2 \times 10^{3}\right]$, and within this domain, we fixed 8 values of areas: $(25,35,50,400,625,900,1200,2000)$, and 28 values of velocities: $(25,50,75,100,200$, $300,400,500,600,700,800,900,1000,1200,1400,1600,1800,3000,3200,5400,6400$, $9000,11000,16000,18000,22400,25200,40000)$. These values were chosen with the aim 
of capturing the characteristics of the surface at low and high parameter values. Using periodic boundary conditions, we then produced 10 simulations for each corresponding pair of parameters and finally computed the contact averages. The simulations were run in NetLogo ${ }^{1}$ Railsback \& Grimm (2012) and the code is available upon request to K. Snyder. ${ }^{2}$ It is well known that the average number of contacts observed during a fixed period of time should increase with larger velocities and decrease with larger areas, therefore suggesting a relation of the form

$$
c=\frac{a v^{p}}{A^{q}}
$$

where $p, q$ and $a$ are constants. This expression can then be rewritten in terms of the dimensionless quantities $\Pi_{1}$ and $\Pi_{2}$,

$$
c=\overbrace{\frac{a}{n_{m *}^{q} t_{*}^{p} R_{*}^{2 q-p}}}^{K} \Pi_{2}^{p-q} \Pi_{1}^{q}=K\left(\frac{v t}{R}\right)^{p-q}\left(\frac{v t R n_{m}}{A}\right)^{q} .
$$

Using least squares to fit relation (3) to the averages of the data points collected gave approximate values of $p \approx 1, q \approx 1$ and $K=1.2887$. Denoting with $n_{f}$ the number of females and with $C$ the total averaged number of encounters that females have with males, i.e. $C=c n_{f}$, gives

$$
C=K \frac{v t R}{A} n_{m} n_{f}=K v t R A x y,
$$

where $x$ and $y$ are the densities of males and females, respectively. The right-hand term in (4) is traditionally obtained from the theory of molecular collisions, also known as the ideal gas model. That theory provides a constant value of $K=4 / \pi=1.2732 \ldots$, which is in good agreement with the value obtained through our simulations (the relative error is less than $2 \%$ ).

\subsection{Mating encounters}

We generate different data by repeating the simulations of individual movement with the same assumptions as above but counting at most one mating encounter per female at each time step ruling out simultaneous mating. Fitting the model to the new data produces $p \approx 1, q \approx 1$ and $K \approx .1231$, which is less than $10 \%$ of the previous $K$ value.

Our interest now is to include changes in movement direction at every step in time, depending on the previous direction rather than restricting individual movement to straight lines as in the assumptions. A limitation in the theoretical ideal gas model, as traditionally conceived, is that it does not capture the effects of varying correlation in individuals' movement (i.e. the autocorrelation of the directions of subsequent individual moves) on contact rates. From the dimensional analysis, however, we conclude that the degree of correlation should appear as a functional dependence between the value $K$ and the range of possible directions for individuals' movement, i.e. $K=K(\theta)$, where at each time step each individual changes to a direction chosen uniformly at random from $[-\theta, \theta]$, $0 \leq \theta \leq \pi$, independently of the other individuals. Repeating the numerical experiments but now allowing individuals to change movement direction with different degrees of correlation, we compute the average of $K$ for different values of $\theta$. Unsurprisingly, these values appear almost constant, as first pointed out by Skellam (1958) for the classical 

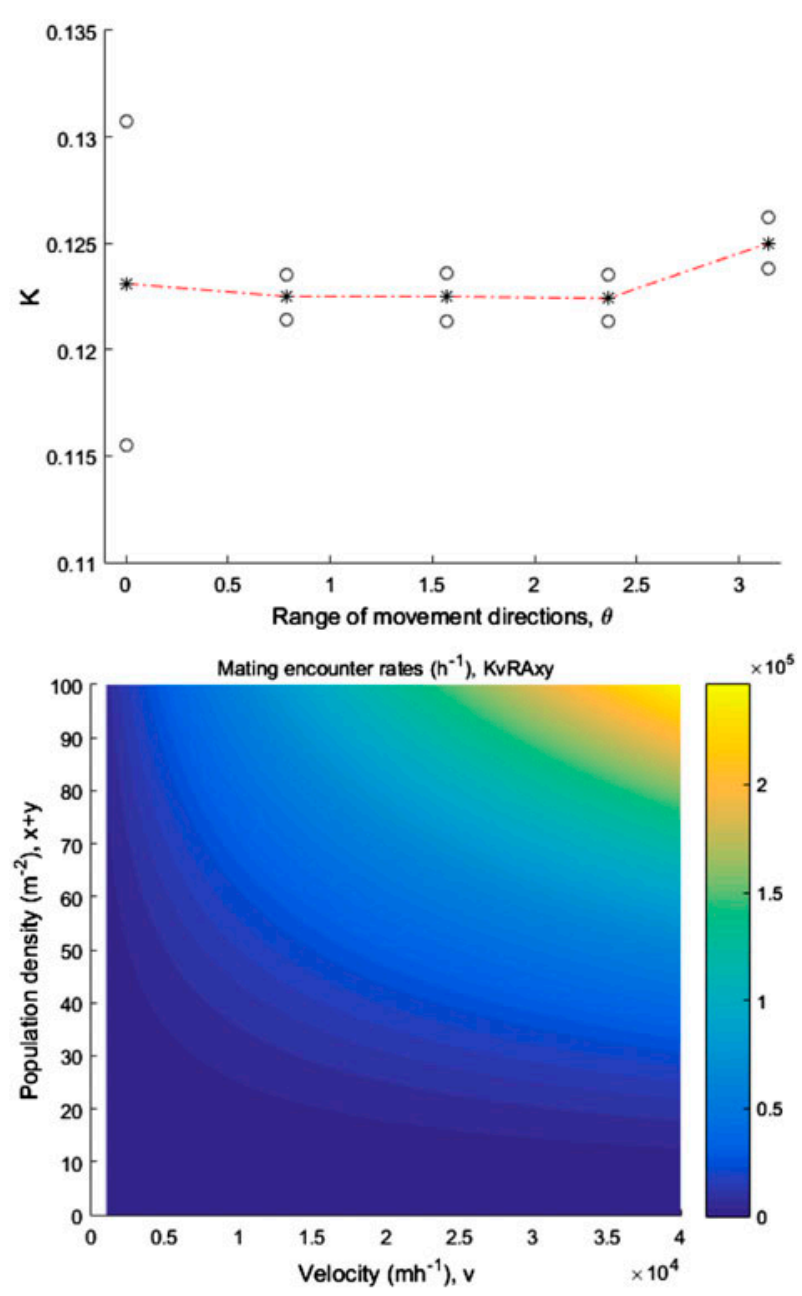

Figure 1. Top: Averaged values of $K$ obtained from observations where particles change their direction at each step in an angle chosen randomly from $[-\theta, \theta]$. The averages (stars joined with dash/dot curve) do not differ significantly from the mean $\bar{K}=.1231$. For each angle $\theta$, the circles mark the values of $K_{+}$ and $K_{-}$, which are the constants obtained by fitting the relation (3) to the original average data points \pm standard deviation, respectively. Bottom: Mating encounter rates (encounters/h) for moving individuals as function of the population density (individuals $/ \mathrm{m}^{2}$ ) and velocity $(\mathrm{m} / \mathrm{h}$ ). For illustration purposes, the detection radius $R$ was fixed and chosen equal to $.02 \mathrm{~m}$ and the velocities are in the range from 1 to $40 \mathrm{Km} / \mathrm{h}$, which includes estimates for several insect species, see Table 1 . The area $A$ is one square meter.

mass action, see Figure 1 (top). This information can thus be used to approximate average mating encounter rates for individuals, given estimates of velocities, see Figure 1 (bottom). We emphasize, however, that the assumptions made on the movement constitute a rough simplification of reality: males and females do not necessarily move at the same speed and the details of mating mechanisms have been deliberately left out.

To assess the variability due to movement correlation on the parameter $K$, we re-fit Equation (3) for different values of $\theta$ to two different data-sets defined by taking the simulated data at each corresponding point of the $v-A$ plane and then computing average+standard deviation and average-standard deviation. With these sets of points, 
Table 1. Average velocities for sustained flight in some insects, Nachtigall (1974).

\begin{tabular}{lc}
\hline Insect & Flight velocity $(\mathrm{Km} / \mathrm{h})$ \\
\hline Mayflies, small field grasshoppers & 1.8 \\
Bumblebees, rose chafers & 3.0 \\
Anopheles (malaria mosquitoes) & 3.2 \\
Stag beetle, damselfly, Ammophila (a fossorial wasp) & 5.4 \\
Housefly & 6.4 \\
Cockchafer, cabbage white butterfly, garden wasp & 9.0 \\
Blowfly & 11.0 \\
Desert locust & 16.0 \\
Hummingbird hawk moth & 18.0 \\
Honeybee, horsefly & 22.4 \\
Aeshna (a dragonfly), hornet & 25.2 \\
Anax (a dragonfly) & 30.0 \\
Deer botfly & 40.0 \\
\hline
\end{tabular}

we obtained two new constants (that depend on $\theta$ ) and denoted by $K_{+}$and $K_{-}$. The data suggested that for values of $\theta$ close to zero, the differences are consistently larger, giving larger values for $K_{+}$and $K_{-}$(See Figure 1). But with increasing $\theta$, although the movement is more irregular, large variability in the values of $K_{+}$and $K_{-}$is absent. Our interpretation of this outcome is that, although individual movement is apparently more convoluted for larger $\theta$, at each trial the (stochastic) process is the same. On the contrary, for values of $\theta$ close to zero, the initial random directions for each individual define paths that look like the deterministic trajectories $(\theta=0)$ in the domain (in this case, a torus). Those might differ completely each time the experiment is repeated because of the randomization of initial directions. This fact was found to be independent whether the boundary conditions are periodic or reflective after running additional simulations for the latter.

\section{Effects of environmental stochasticity}

The rate at which offspring are generated by a two-sex population in real scenarios is likely to be subject to random fluctuations due to environmental factors, like rainfall and temperature, but with sensitivity that is species specific. If mass action is chosen to model mating encounters, variability on the parameter $K$ will appear as consequence of those fluctuations. This translates directly into variability of birth and death rates. As an illustration, let us initially consider the simplest deterministic population model ephemeral mating interactions,

$$
\begin{aligned}
& x^{\prime}=-\mu x+\frac{1}{2} P(x, y), \\
& y^{\prime}=-\mu y+\frac{1}{2} P(x, y),
\end{aligned}
$$

where $x$ and $y$ are the densities of males and females, respectively, $P(x, y)$ is a (symmetric) birth rate with even sex ratio for all births and $\mu$ is the death rate that is assumed equal for both sexes. Suppose also that the initial sex ratio is $1: 1$. It is natural that we would like to use the law of mass action for $P(x, y)$, i.e. proportional to $x y$, but as D.G. Kendall first pointed out, this leads to solutions that blow up in finite time (Kendall, 1949). One way, this trouble can be fixed by taking into consideration the average refractory time $\tau$ of 


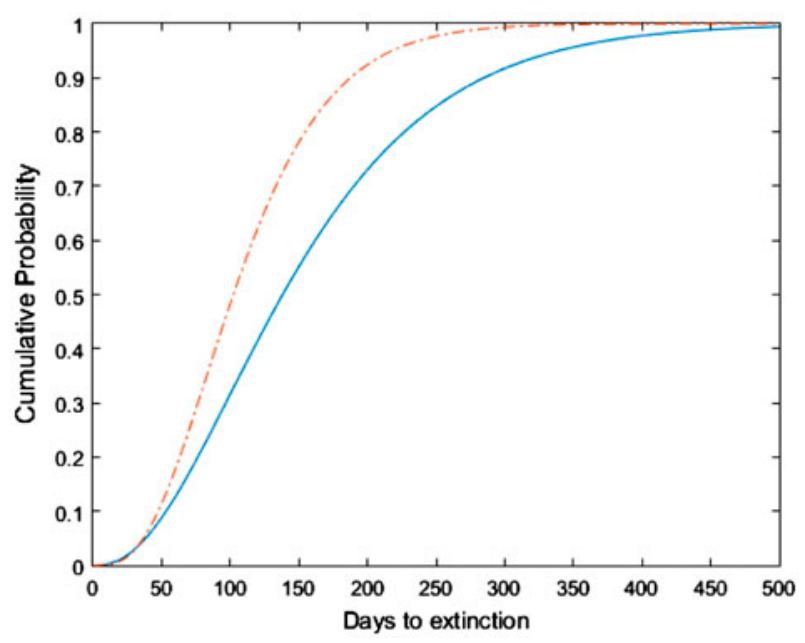

Figure 2. Cumulative probability of extinction (conditioned to extinction) obtained from the stochastic model defined in Section 3. The effect of variability on the number of contacts, $K \sim \operatorname{Normal}\left(\bar{K}, \sigma^{2}\right)$, is shown for different values of $\sigma^{2}=0$ (continuous line), and .1 (dash-dots). The initial population is taken equal to half the Allee threshold. The simulations suggest that an increasing variance in the random fluctuations corresponding to mating encounters pushes the probability mass to the left, i.e. extinction is likely to happen sooner than otherwise expected. The parameters for the simulations were chosen arbitrarily $1 / \mu=10$ (days), $v=1.33(\mathrm{Km} / \mathrm{h}), R=.02(\mathrm{~m})$ and $b=3$, although they resemble the characteristics in some relatively small insects. The value $p=.01$, which in practice depends on the complexity of the mating mechanisms, was also set arbitrarly.

females (Bazykin, 1998), during which a female avoids further sexual encounters just after successfully mating with a male. Let $r$ denote the rate of mating encounters per female, $r=K v R x=\alpha x$, then $1 / r$ is the average time between encounters for one female. If the population densities are low enough so that $1 / r$ is very much more than $\tau$, then the population growth will depend on the number of successful mating encounters made. In this case, the average progeny produced by a female has to saturate when $1 / r \downarrow \tau$ as the density of males increases. Therefore, the average birth rate per female is more conveniently approximated with $b p \times \alpha x \times \frac{1}{N+x}$, where $b$ is the average number of offspring per female per encounter that survive to adulthood, $p$ is the probability that an encounter produces offspring and $N$ is the average male population density at which half of the females are able to reproduce. This can be seen by making $x=N$, so the average birth rate per female is $b p \alpha / 2$ or equivalently that half of the females produce offspring at the per capita rate $b p \alpha$. For simplicity, we have set $N=1$, suggested by the reasonable approximation of the mean number of pairs formed at low densities (Gordillo, 2015). Given that initially we assumed a 1:1 sex ratio, the whole population dynamics can be described by the equation

$$
z^{\prime}=-\mu z+P(z / 2, z / 2)=-\mu z+b p \alpha \frac{z^{2}}{2(2+z)}=\frac{\gamma z(z-2 \mu / \gamma)}{2+z}
$$

where $z=x+y$ and $\gamma=(b p \alpha-2 \mu) / 2>0$. Thus, if $z \ll 2$, the population growth will approximately correspond to a mass action regime while if $z \gg 2$, it grows exponentially. More precisely, $z^{\prime}<0$ when $z<2 \mu / \gamma$, giving rise to positive density dependence with a critical population size. 
We construct a stochastic version of the model by seeing the birth and death rates as stochastic rates (Gillespie, 1977); that is, we integrate demographic random fluctuations by considering only the two types of events, birth and death of individuals, happening at exponentially distributed times with the overall rate $\Psi$ given by

$$
\Psi=\frac{b p \alpha z^{2}}{2(2+z)}+\mu z .
$$

At a given time, a death happens with probability $q=\mu z / \Psi$, or a birth with probability $1-$ $q$. In addition, environmental stochasticity is introduced by letting $K$ fluctuate randomly, with $K \sim \operatorname{Normal}\left(\bar{K}, \sigma^{2}\right)(\bar{K}=.1231)$. The effects of varying $\sigma^{2}$ are displayed in Figure 2, which shows the cumulative probabilities of extinction up to a fixed time, conditioned on extinction. The initial population density is taken at half of the Allee threshold value. The results suggest that an increasing variance in the random fluctuations associated with the mating encounters would increase the probability of earlier extinction events.

\section{Discussion}

Mating processes in two-sex insect populations are species specific and generally involve vast complexity (Bonduriansky, 2001; Choe \& Crespi, 1997). This necessitates an approximated description of the non-linear process for mating encounters. Mass action has been continuously used for this end, even in cases with convoluted mating mechanisms (Courchamp et al., 2008). The multiplicative constant for the mating encounter rate might be difficult to estimate in many real applications. The novelty in this note is that, under general assumptions, we have approximated the value of that constant with the use of dimensional reduction and simulated data. Before now, this constant has not been computed by such analytical arguments.

The dimensional reduction approach also offers a way to examine the effects of correlation in the movement. The simulations suggest that in general, the variances of the values of $K$ are small and similar when compared among results obtained with different degrees of correlation. Thus, the value $\bar{K}$ can be used as the multiplicative constant in the expression for the encounter rate, $\bar{K} v R A x y$, independently of movement correlation. Relative larger variance values observed for highly correlated movement ( $\theta$ close to zero) are attributed to the boundary conditions in the simulations. We remark that the programme used to produce the data only counts ephemeral encounters and does not consider further association between paired individuals, as well as other complexities involved in mating mechanisms.

While our analysis assumes at most one mating encounter per female at each time step, the approach may be generalized to systems where individuals must spend more or less time between mating encounters. For the purposes of modelling sex role evolution, $\mathrm{H}$. Kokko and M. Jennions presented in Kokko and Jennions (2008) a scheme that accounts for time delays following encounters when individuals cannot mate. This framework could be adopted to modify and extend our work.

Small random fluctuations in the number of mating encounters can appear nevertheless, caused by non-permanent random environmental changes. These effects might alter substantially the encounter rate (now random). As an illustration, we simulated a basic 
stochastic birth and death process to obtain the probability distribution of the time to extinction, conditioned on extinction. The model has a deterministic analogue for which extinction due to Allee effects is possible. The simulations suggest that small fluctuations on the parameter $\bar{K}$ might induce relatively large increments on the (conditioned) cumulative probability of extinction, see Figure 2. For conservation or pest control efforts in which it is critical to assess how rapidly a population might become extinct, the approximation for $\bar{K}$ presented here might help shape more accurate quantitative predictions based on the law of mass action.

We would like to emphasize that, after all, mass action is a highly idealized model where physical and biological details are overlooked, including those corresponding to mating mechanisms. For instance, velocities associated with aerial mating in insects may be sensitive to the relative mass of flight muscle (Dudley, 2000), while (the radius of) attraction may depend on chemical, visual or auditory signals (Resh \& Cardé, 2009; Roelofs, 1995). Trying to introduce these species-specific details into the mass action scheme could be a challenging task that we have not considered. In the case that velocity and radius of attraction were both scaled to individual total mass, for example, it would be possible to insert these relations into the main formulae as long as the assumptions for the mass action still hold. If the case is that individual mass implies non-negligible (relative) size, then the simulations cannot be used as we set them, and new computational experiments together with a re-assessment of the whole model have to be done. In contrast to the crude assumptions made for mass action, we refer the reader to the interesting papers (Gurarie \& Ovaskainen, 2011, 2013), where the authors present a refined continuous timecontinuous space theoretical framework that shows how encounter rates depend on the interplay of spatial distributions, scales of movement, individuals densities and inherent dynamics. However, we believe the simple model presented here can inform the theoretical modelling of ephemeral (short-lived) mating encounters for insects, important in pest control management (Boukal \& Berec, 2008; Fauvergue, 2013; Gordillo, 2015).

\section{Notes}

1. https://ccl.northwestern.edu/netlogo/.

2. Katherine.Snyder@usu.edu.

\section{Acknowledgements}

The authors are deeply grateful to P. Greenwood, P.M. Waser and J.M.C. Hutchinson and two anonymous reviewers for the extensive and useful comments, and suggestions on previous versions of the manuscript.

\section{Disclosure statement}

No potential conflict of interest was reported by the authors.

\section{References}

Barenblatt, G. (2006). Scaling. Cambridge: Cambridge Text in Applied Mathematics, Cambridge University Press. 
Bartumeous, F., Catalan, J., Viswanathan, G., Raposo, E., \& da Luz, M. (2008). The influence of turning angles on the success of non-oriented animal searches. Journal of Theoretical Biology, 252, 43-55.

Bazykin, A. (1998). Nonlinear dynamics of interacting populations. Singapore: World Scientific Publishing.

Bonduriansky, R. (2001). The evolution of male mate choice in insects: A synthesis of ideas and evidence. Biological Reviews, 76, 305-339.

Boukal, D., \& Berec, L. (2008). Modelling mate-finding Allee effects and population dynamics, with applications in pest control. Population Ecology, 51, 445-458.

Caswell, H. (2001). Matrix population models. Sutherland, MA: Sinauer Associates.

Choe, J., \& Crespi, B. (1997). Mating systems in insects and arachnids. Cambridge: Cambridge University Press.

Codling, E., Plank, M. J., \& Benhamou, S. (2008). Random walk models in biology (review). The Journal of the Royal Society Interface, 5, 813-834.

Courchamp, F., Berec, L., \& Gascoigne, J. (2008). Allee effects in ecology and conservation. New York, NY: Oxford University Press.

Dudley, R. (2000). The biomechanics of insect flight. Princeton, NJ: Princeton University Press.

Fauvergue, X. (2013). A review of mate-finding allee effects in insects: From individual behavior to population management. Entomologia Experimentalis et Applicata, 146, 79-92.

Gillespie, D. (1977). Exact simulation of coupled chemical reactions. The Journal of Physical Chemistry, 81, 2340-2361.

Gordillo, L. (2015). Modeling ephemeral mating encounters in insects: The emergence of matefinding Allee effects and applications to theoretical models of sterile release. Theoretical Population Biology, 104, 10-16.

Gurarie, E., \& Ovaskainen, O. (2011). Characteristic spatial and temporal scales unify models of animal movement. The American Naturalist, 178, 113-123.

Gurarie, E., \& Ovaskainen, O. (2013). Towards a general formalization of encounter rates in ecology. Theoretical Ecology, 6, 189-202.

Heesterbeek, H. (2005). The law of mass-action in epidemiology: A historical perspective. In B. B. K. Cuddington (Ed.), Ecological paradigms lost, routes of theory change (pp. 81-105). Burlington, MA: Elsevier Academic Press.

Hutchinson, J., \& Waser, P. (2007). Use, misuse and extensions of "ideal gas" models of animal encounter. Biological Reviews, 82, 335-359.

James, A., Pitchford, J., \& Plank, M. (2010). Efficient or innacurate? Analytical and numerical modelling of random search strategies. Bulletin of Mathematical Biology, 72, 896-913.

James, A., Plank, M., \& Brown, R. (2008). Optimizing the encounter rate in biological interactions: Ballistic vs. lévy vs. brownian strategies. Physical Review E, 78, 51128.

Kauzmann, W. (2012). Kinetic theory of gases. Mineola, NY: Dover Publications.

Kendall, D. (1949). Stochastic processes and population growth. Journal of the Royal Statistical Society, Series B, 11, 230-282.

Kokko, H., \& Jennions, M. (2008). Parental investment, sexual selection and sex ratios. Journal of Evolutionary Biology, 21, 919-948.

Logan, J. (2006). Applied mathematics (3rd ed.). Hoboken, NJ: Wiley.

Lotka, A. (1925). Elements of physical biology. Baltimore: Williams \& Wilkins Company.

McKendrick, A. (1912). The rise and fall of epidemics. Paludism, 1, 54-66.

Nachtigall, W. (1974). Insects in flight. A glimpse behind the scenes in biophysical research. London: George Allen \& Unwin.

Railsback, S., \& Grimm, V. (2012). Agent-based and individual-based modeling. Princeton, NJ: Princeton University Press.

Resh, V., \& Cardé, R. (2009). Encyclopedia of insects (2nd ed.). Burlington, MA: Elsevier.

Roelofs, W. (1995). Chemistry of sex attraction. Proceedings of the National Academy Science USA, 92, 44-49.

Skellam, J. (1958). The mathematical foundations underlying the use of line transects in animal ecology. Biometrics, 14, 385-400. 
Turchin, P. (1998). Quantitative analysis of movement: Measuring and modeling population redistribution in animals and plants. Sunderland: Sinauer.

Voit, E., Martens, H., \& Omholt, S. (2015). 150 years of the mass action law. PLoS Computational Biology, 11, e1004012. 